TRANSACTIONS OF THE

AMERICAN MATHEMATICAL SOCIETY

Volume 354, Number 1, Pages 205-217

S 0002-9947(01)02867-7

Article electronically published on August 29, 2001

\title{
THE MULTIPLIER IDEALS OF A SUM OF IDEALS
}

\author{
MIRCEA MUSTAŢĂ
}

\begin{abstract}
We prove that if $\underline{\mathbf{a}}, \underline{\mathbf{b}} \subseteq \mathcal{O}_{X}$ are nonzero sheaves of ideals on a complex smooth variety $X$, then for every $\gamma \in \mathbb{Q}+$ we have the following relation between the multiplier ideals of $\underline{\mathbf{a}}, \underline{\mathbf{b}}$ and $\underline{\mathbf{a}}+\underline{\mathbf{b}}$ :

$$
\mathcal{I}(X, \gamma \cdot(\underline{\mathbf{a}}+\underline{\mathbf{b}})) \subseteq \sum_{\alpha+\beta=\gamma} \mathcal{I}(X, \alpha \cdot \underline{\mathbf{a}}) \cdot \mathcal{I}(X, \beta \cdot \underline{\mathbf{b}}) .
$$

A similar formula holds for the asymptotic multiplier ideals of the sum of two graded systems of ideals.

We use this result to approximate at a given point arbitrary multiplier ideals by multiplier ideals associated to zero dimensional ideals. This is applied to compare the multiplier ideals associated to a scheme in different embeddings.
\end{abstract}

\section{INTRODUCTION}

Let $X$ be a smooth complex variety. To a nonzero quasi-coherent sheaf of ideals $\underline{\mathbf{a}}$ on $X$ one can associate a sequence of ideals called the multiplier ideals of $\underline{\mathbf{a}}$, which depend on a rational parameter. The behaviour of these ideals encodes in a subtle way the properties of the singularities of $V(\underline{\mathbf{a}})$. Introduced first in the analytic context in the work of Demailly, Nadel, Siu and others, multiplier ideals have recently found surprising applications in algebraic geometry (see [Ein], Siu], [Ka1], [Ka2], [EL], [ELS]).

Here is the definition. Suppose that $f: X^{\prime} \longrightarrow X$ is a $\log$ resolution of $(X, V(\underline{\mathbf{a}}))$, i.e., $f$ is proper and birational, $X^{\prime}$ is smooth, and $f^{-1} V(\underline{\mathbf{a}})=D$ is a divisor with simple normal crossings. If $K_{X^{\prime} / X}$ is the relative canonical divisor of $f$, the multiplier ideal of $\underline{\mathbf{a}}$ with coefficient $\alpha \in \mathbb{Q}_{+}$is

$$
\mathcal{I}(X, \alpha \cdot \underline{\mathbf{a}})=f_{*} \mathcal{O}_{X^{\prime}}\left(K_{X^{\prime} / X}-[\alpha D]\right) .
$$

Here $[\cdot]$ denotes the integral part function.

In general one can expect that algebraic properties of the multiplier ideals are related to the behaviour of linear systems and singularities of algebraic varieties. For example, the following subadditivity relation is proved in [DEL]:

$$
\mathcal{I}(X, \alpha \cdot(\underline{\mathbf{a}} \cdot \underline{\mathbf{b}})) \subseteq \mathcal{I}(X, \alpha \cdot \underline{\mathbf{a}}) \cdot \mathcal{I}(X, \alpha \cdot \underline{\mathbf{b}}) .
$$

This is applied in [DEL] to prove a theorem of Fujita on the volume of a big divisor and in [ELS] to show a uniform behaviour of the symbolic powers of an ideal.

The main result of this paper is an analogous formula for the sum of two ideals.

Received by the editors March 1, 2001.

2000 Mathematics Subject Classification. Primary 14B05; Secondary 14 F17.

Key words and phrases. Multiplier ideals, log resolutions, monomial ideals.

(C)2001 American Mathematical Society 
Theorem 0.1. If $X$ is a smooth variety and $\underline{\mathbf{a}}, \underline{\mathbf{b}} \subseteq \mathcal{O}_{X}$ are nonzero sheaves of ideals, then for every $\gamma \in \mathbb{Q}_{+}$we have

$$
\mathcal{I}(X, \gamma \cdot(\underline{\mathbf{a}}+\underline{\mathbf{b}})) \subseteq \sum_{\alpha+\beta=\gamma} \mathcal{I}(X, \alpha \cdot \underline{\mathbf{a}}) \cdot \mathcal{I}(X, \beta \cdot \underline{\mathbf{b}}) .
$$

Note that the sum has finitely many distinct terms. The above statement admits a generalization to the case of two graded systems of ideals. Recall that a graded system of ideals $\underline{\mathbf{a}}_{\mathbf{.}}=\left(\underline{\mathbf{a}}_{m}\right)_{m \geq 0}$ on $X$ is a sequence of nonzero ideals such that $\underline{\mathbf{a}}_{0}=\mathcal{O}_{X}$ and $\underline{\mathbf{a}}_{p} \cdot \underline{\mathbf{a}}_{q} \subseteq \underline{\mathbf{a}}_{p+q}$, for every $p$ and $q$. It is proved in [ELS] that the set $\left\{\mathcal{I}\left(X, \alpha / q \cdot \underline{\mathbf{a}}_{p q}\right)\right\}_{q \geq 1}$ has a unique maximal element, the asymptotic multiplier ideal $\mathcal{I}\left(X, \alpha \cdot\left\|\underline{\mathbf{a}}_{p}\right\|\right)$.

Suppose now that we have two graded systems of ideals $\underline{\mathbf{a}}_{\mathbf{}}$ and $\underline{\mathbf{b}}_{\mathbf{.}}$. Their sum $\underline{\mathbf{c}}_{\bullet}=\underline{\mathbf{a}}_{\bullet}+\underline{\mathbf{b}}_{\mathbf{\bullet}}$, defined by $\underline{\mathbf{c}}_{m}=\sum_{i+j=m} \underline{\mathbf{a}}_{i} \underline{\mathbf{b}}_{j}$, is again a graded system of ideals. With these definitions we have the following:

Theorem 0.2. Let $X$ be a smooth variety and $\underline{\mathbf{a}}$. and $\underline{\mathbf{b}}$. two graded systems of ideals on $X$ and $\underline{\mathbf{c}}$. their sum. For every $\gamma \in \mathbb{Q}_{+}$and every $p \geq 1$, we have

$$
\mathcal{I}\left(X, \gamma \cdot\left\|\underline{\mathbf{c}}_{p}\right\|\right) \subseteq \sum_{\alpha+\beta=\gamma} \mathcal{I}\left(X, \alpha \cdot\left\|\underline{\mathbf{a}}_{p}\right\|\right) \cdot \mathcal{I}\left(X, \beta \cdot\left\|\underline{\mathbf{b}}_{p}\right\|\right) .
$$

Note that Theorem 0.1 can be obtained from Theorem 0.2 by taking the systems $\underline{\mathbf{a}}$. and $\underline{\mathbf{b}}$. to be given by the powers of $\underline{\mathbf{a}}$ and $\underline{\mathbf{b}}$, respectively.

As an application of Theorem 0.1, in the second part of the paper we show that general multiplier ideals can be approximated at each point by multiplier ideals associated to zero dimensional ideals (see Theorem 2.1 for the precise statement). This is then used to compare the multiplier ideals associated to a scheme in different embeddings. For example, we show that if $\underline{\mathbf{a}} \subseteq \mathcal{O}_{X}$ and $\underline{\mathbf{b}} \subseteq \mathcal{O}_{Y}$ are such that $V(\underline{\mathbf{a}}) \simeq V(\underline{\mathbf{b}})$, then this isomorphism maps $\mathcal{I}(X, \alpha \cdot \underline{\mathbf{a}}) \cdot \mathcal{O}_{V(\underline{\mathbf{a}})}$ to $\mathcal{I}(Y, \beta \cdot \underline{\mathbf{b}}) \cdot \mathcal{O}_{V(\underline{\mathbf{b}})}$ if $\operatorname{dim} X-\alpha=\operatorname{dim} Y-\beta$.

We give below the idea of the proof of the main results. For simplicity, we consider only the case of Theorem 0.1. The proof of Theorem 0.2 follows from a similar, but more technical statement, which can be proved in an analogous way (see Theorem 1.1).

The first step is to use the Restriction Theorem for the diagonal embedding $\Delta: X \hookrightarrow X \times X$ to reduce the statement of Theorem 0.1 to a result on $X \times X$. This is the particular case $X=Y$ in the following:

Theorem 0.3. Let $X$ and $Y$ be smooth varieties and $\underline{\mathbf{a}} \subseteq \mathcal{O}_{X}$ and $\underline{\mathbf{b}} \subseteq \mathcal{O}_{Y}$ nonzero sheaves of ideals. If $p: X \times Y \longrightarrow X$ and $q: X \times Y \longrightarrow Y$ are the canonical projections, then for every $\gamma \in \mathbb{Q}_{+}$we have

$$
\mathcal{I}\left(X \times Y, \gamma \cdot\left(p^{-1} \underline{\mathbf{a}}+q^{-1} \underline{\mathbf{b}}\right)\right)=\sum_{\alpha+\beta=\gamma} p^{-1} \mathcal{I}(X, \alpha \cdot \underline{\mathbf{a}}) \cdot q^{-1} \mathcal{I}(Y, \beta \cdot \underline{\mathbf{b}}) .
$$

The next step is to reduce Theorem 0.3 by taking log resolutions to the case when $\underline{\mathbf{a}}$ and $\underline{\mathbf{b}}$ are ideals defining divisors with simple normal crossings. However, as it stands, the right-hand side of equation (3) does not behave well with respect to push-forward. Therefore we first prove a lemma showing that, in fact, with the above notation, we have

$$
\sum_{\alpha+\beta=\gamma} p^{-1} \mathcal{I}(X, \alpha \cdot \underline{\mathbf{a}}) \cdot q^{-1} \mathcal{I}(Y, \beta \cdot \underline{\mathbf{b}})=\bigcap_{\alpha+\beta=\gamma}\left(p^{-1} \mathcal{I}(X, \alpha \cdot \underline{\mathbf{a}})+q^{-1} \mathcal{I}(Y, \beta \cdot \underline{\mathbf{b}})\right) .
$$


Using this expression, we can reduce ourselves to the case of divisors with simple normal crossings. Note, however that $p^{-1} \underline{\mathbf{a}}+q^{-1} \underline{\mathbf{b}}$ has codimension two. On the other hand, locally in the étale topology $\underline{\mathbf{a}}$ and $\underline{\mathbf{b}}$ are monomial ideals and therefore so is $p^{-1} \underline{\mathbf{a}}+q^{-1} \underline{\mathbf{b}}$. The equality in Theorem 0.3 follows now using the explicit description of multiplier ideals of monomial ideals due to Howald (see [Ho]).

Acknowledgements. We are grateful to Lawrence Ein and Rob Lazarsfeld for their encouragement and for their comments on this work. In particular, the extension of Theorem 0.1 to asymptotic multiplier ideals was suggested to us by Rob Lazarsfeld.

\section{The multiplier ideals of A SUM OF IDEALS}

We work over the field of complex numbers. All sheaves of ideals are assumed to be quasi-coherent. The basic results on multiplier ideals that will be used can be found in [Ein], [DEL] and [ELS] (see also [La] for a thorough presentation of the subject).

The following theorem is the main technical result of this section. It easily implies Theorems 0.1 and 0.2

Theorem 1.1. Let $X$ and $Y$ be smooth varieties and $p: X \times Y \longrightarrow X$ and $q: X \times Y \longrightarrow Y$ the canonical projections. Suppose that $\underline{\mathbf{a}}_{i} \subseteq \mathcal{O}_{X}, \underline{\mathbf{b}}_{i} \subseteq \mathcal{O}_{Y}$ are nonzero sheaves of ideals for $1 \leq i \leq m$ or $i=n$. If for every $1 \leq i \leq m$ we have $i \mid n$ and $\underline{\mathbf{a}}_{i}^{n / i} \subseteq \underline{\mathbf{a}}_{n}$ and $\underline{\mathbf{b}}_{i}^{n / i} \subseteq \underline{\mathbf{b}}_{n}$, then

$$
\begin{gathered}
\mathcal{I}\left(X \times Y, \gamma / m \cdot\left(p^{-1} \underline{\mathbf{a}}_{m}+\sum_{i=1}^{m-1} p^{-1} \underline{\mathbf{a}}_{i} \cdot q^{-1} \underline{\mathbf{b}}_{m-i}+q^{-1} \underline{\mathbf{b}}_{m}\right)\right) \\
\subseteq \sum_{\alpha+\beta=\gamma} p^{-1} \mathcal{I}\left(X, \alpha / n \cdot \underline{\mathbf{a}}_{n}\right) \cdot q^{-1} \mathcal{I}\left(Y, \beta / n \cdot \underline{\mathbf{b}}_{n}\right),
\end{gathered}
$$

for every $\gamma \in \mathbb{Q}_{+}$.

We first prove the following lemma.

Lemma 1.2. Let $X$ and $Y$ be smooth varieties, $p: X \times Y \longrightarrow X$ and $q: X \times$ $Y \longrightarrow Y$ the canonical projections, and $\underline{\mathbf{a}} \subseteq \mathcal{O}_{X}$ and $\underline{\mathbf{b}} \subseteq \mathcal{O}_{Y}$ nonzero sheaves of ideals. For every rational number $\gamma \geq 0$, we have

$$
\sum_{\alpha+\beta=\gamma} p^{-1} \mathcal{I}(X, \alpha \cdot \underline{\mathbf{a}}) \cdot q^{-1} \mathcal{I}(Y, \beta \cdot \underline{\mathbf{b}})=\bigcap_{\alpha+\beta=\gamma}\left(p^{-1} \mathcal{I}(X, \alpha \cdot \underline{\mathbf{a}})+q^{-1} \mathcal{I}(Y, \beta \cdot \underline{\mathbf{b}})\right) .
$$

Proof. In order to prove " $\subseteq$ ", we have to show that if $\alpha+\beta=\alpha_{1}+\beta_{1}=\gamma$, then

$$
p^{-1} \mathcal{I}(X, \alpha \cdot \underline{\mathbf{a}}) \cdot q^{-1} \mathcal{I}(Y, \beta \cdot \underline{\mathbf{b}}) \subseteq p^{-1} \mathcal{I}\left(X, \alpha_{1} \cdot \underline{\mathbf{a}}\right)+q^{-1} \mathcal{I}\left(Y, \beta_{1} \cdot \underline{\mathbf{b}}\right) .
$$

It is clear that we must have either $\alpha \geq \alpha_{1}$ or $\beta \geq \beta_{1}$. In the first case we have $\mathcal{I}(X, \alpha \cdot \underline{\mathbf{a}}) \subseteq \mathcal{I}\left(X, \alpha_{1} \cdot \underline{\mathbf{a}}\right)$ and the above inclusion follows. The other case is similar.

In order to prove the reverse inclusion, we may assume that $X$ and $Y$ are affine and let $R=\mathcal{O}(X)$ and $S=\mathcal{O}(Y)$. We identify the multiplier ideals with their global sections.

We choose bases for $R$ and $S$ which are compatible with the filtrations induced by the respective multiplier ideals, as follows. Note that the set of multiplier ideals of $\underline{\mathbf{a}}$ with coefficient $p, 0 \leq p \leq \gamma$ is finite and

$$
\mathcal{I}(X, p \cdot \underline{\mathbf{a}}) \subseteq \mathcal{I}\left(X, p^{\prime} \cdot \underline{\mathbf{a}}\right),
$$


if $p>p^{\prime}$. Therefore we can choose index sets $I_{\lambda}$, possibly empty, for $0 \leq \lambda \leq \gamma$, and elements $e_{\lambda \mu} \in R$, for $0 \leq \lambda \leq \gamma$ and $\mu \in I_{\lambda}$ such that for every $p \leq \gamma$, a basis over $\mathbb{C}$ for $\mathcal{I}(X, p \cdot \underline{\mathbf{a}})$ is given by $\left\{e_{\lambda \mu} \mid \lambda \leq \gamma-p, \mu \in I_{\lambda}\right\}$.

We consider an analogous set of elements $f_{\lambda \mu} \in S$, with $0 \leq \lambda \leq \gamma$ and $\mu \in J_{\lambda}$, satisfying the corresponding property with respect to the multiplier ideals of $\underline{\mathbf{b}}$.

$\mathrm{A}$ basis in $p^{-1} \mathcal{I}(X, \alpha \cdot \underline{\mathbf{a}}) \cdot q^{-1} \mathcal{I}(Y, \beta \cdot b)$ is given by

$$
\left\{e_{\lambda \mu} \otimes f_{\lambda^{\prime} \mu^{\prime}} \mid \lambda \leq \gamma-\alpha, \lambda^{\prime} \leq \gamma-\beta, \mu \in I_{\lambda}, \mu^{\prime} \in J_{\lambda^{\prime}}\right\} .
$$

Therefore a basis in $\sum_{\alpha+\beta=\gamma} p^{-1} \mathcal{I}(X, \alpha \cdot \underline{\mathbf{a}}) \cdot q^{-1} \mathcal{I}(Y, \beta \cdot \underline{\mathbf{b}})$ is given by

$$
\left\{e_{\lambda \mu} \otimes f_{\lambda^{\prime} \mu^{\prime}} \mid \lambda+\lambda^{\prime} \leq \gamma, \mu \in I_{\lambda}, \mu^{\prime} \in J_{\lambda^{\prime}}\right\} .
$$

It is enough to prove that if

$$
e_{\lambda \mu} \otimes f_{\lambda^{\prime} \mu^{\prime}} \in \bigcap_{\alpha+\beta=\gamma}\left(p^{-1} \mathcal{I}(X, \alpha \cdot \underline{\mathbf{a}})+q^{-1} \mathcal{I}(Y, \beta \cdot \underline{\mathbf{b}})\right),
$$

then $\lambda+\lambda^{\prime} \leq \gamma$. Indeed, the above intersection has a basis given by a subset of $\left\{e_{\lambda \mu} \otimes e_{\lambda^{\prime} \mu^{\prime}}\right\}_{\lambda, \mu, \lambda^{\prime}, \mu^{\prime}}$, because so has each member of the intersection.

For every $\alpha$ and $\beta$ such that $\alpha+\beta=\gamma$, we must have either $\lambda \leq \gamma-\alpha$ or $\lambda^{\prime} \leq \gamma-\beta$. Therefore for every $0 \leq \alpha \leq \gamma$, we have either $\lambda \leq \gamma-\alpha$ or $\lambda^{\prime} \leq \alpha$. This gives $\lambda^{\prime} \leq \gamma-\lambda$ and finishes the proof of the lemma.

The proof of Theorem 1.1 will be based on reduction to the case of monomial ideals. Therefore we first treat this special case in the following lemma.

Lemma 1.3. The statement of Theorem 1.1 is true if $X=\mathbb{A}^{r}, Y=\mathbb{A}^{s}$ and $\underline{\mathbf{a}}_{i} \subseteq \mathbb{C}[X]=\mathbb{C}\left[X_{1}, \ldots, X_{r}\right]$ and $\underline{\mathbf{b}}_{i} \subseteq \mathbb{C}[Y]=\mathbb{C}\left[Y_{1}, \ldots, Y_{s}\right]$ are monomial ideals for all $i$.

Proof. By Lemma 1.2 it is enough to prove that

$$
\begin{gathered}
\mathcal{I}\left(\mathbb{A}^{r} \times \mathbb{A}^{s}, \gamma / m \cdot\left(p^{-1} \underline{\mathbf{a}}_{m}+\sum_{i=1}^{m-1} p^{-1} \underline{\mathbf{a}}_{i} \cdot q^{-1} \underline{\mathbf{b}}_{m-i}+q^{-1} \underline{\mathbf{b}}_{m}\right)\right) \\
\subseteq \bigcap_{\alpha+\beta=\gamma}\left(p^{-1} \mathcal{I}\left(\mathbb{A}^{r}, \alpha / n \cdot \underline{\mathbf{a}}_{n}\right)+q^{-1} \mathcal{I}\left(\mathbb{A}^{s}, \beta / n \cdot \underline{\mathbf{b}}_{n}\right)\right) .
\end{gathered}
$$

If there is $i$ such that $\underline{\mathbf{a}}_{i}=\mathbb{C}[X]$, then $\underline{\mathbf{a}}_{n}=\mathbb{C}[X]$ and therefore $\mathcal{I}\left(X, \alpha \cdot \underline{\mathbf{a}}_{n}\right)=$ $\mathbb{C}[X]$, for every $\alpha$. It follows that the right-hand side of equation (5) is $\mathbb{C}[X, Y]$ and the inclusion is obvious.

We may therefore assume that $\underline{\mathbf{a}}_{i} \neq \mathbb{C}[X]$ for every $i$, and by symmetry, that $\underline{\mathbf{b}}_{i} \neq \mathbb{C}[Y]$, for every $i$. Suppose that for some $u \in \mathbb{N}^{r}$ and $v \in \mathbb{N}^{s}$, we have

$$
X^{u} Y^{v} \in \mathcal{I}\left(\mathbb{A}^{r} \times \mathbb{A}^{s}, \gamma / m \cdot\left(p^{-1} \underline{\mathbf{a}}_{m}+\sum_{i=1}^{m-1} p^{-1} \underline{\mathbf{a}}_{i} \cdot q^{-1} \underline{\mathbf{b}}_{m-i}+q^{-1} \underline{\mathbf{b}}_{m}\right)\right),
$$

but for some $\alpha, \beta \in \mathbb{Q}_{+}$with $\alpha+\beta=\gamma$, we have $X^{u} \notin \mathcal{I}\left(\mathbb{A}^{r}, \alpha / n \cdot \underline{\mathbf{a}}_{n}\right)$ and $Y^{v} \notin \mathcal{I}\left(\mathbb{A}^{s}, \beta / n \cdot \underline{\mathbf{b}}_{n}\right)$.

We use Howald's description for multiplier ideals of monomial ideals in $\underline{\mathrm{Ho}}$. It says that if $I \subset \mathbb{C}[X]$ is a proper nonzero monomial ideal and $P_{I} \subset \mathbb{R}^{r}$ is the convex hull of $\left\{w \in \mathbb{N}^{r} \mid X^{w} \in I\right\}$, then for every $c>0$,

$$
\mathcal{I}\left(\mathbb{A}^{r}, c \cdot I\right)=\left(X^{w} \mid w+e \in \operatorname{Int}\left(c \cdot P_{I}\right)\right) .
$$

Here $e$ denotes the unit vector $(1, \ldots, 1) \in \mathbb{N}^{r}$. 
Our hypothesis on $u$ and $v$ implies that $u+e \notin \operatorname{Int}\left(\alpha / n \cdot P_{\underline{\mathbf{a}}_{n}}\right)$ and $v+f \notin$ $\operatorname{Int}\left(\beta / n \cdot P_{\underline{\mathbf{b}}_{n}}\right)$ (where $\left.f=(1, \ldots, 1) \in \mathbb{N}^{s}\right)$. This means that there are linear maps $\phi: \mathbb{R}^{r} \longrightarrow \mathbb{R}$ and $\psi: \mathbb{R}^{s} \longrightarrow \mathbb{R}$ such that $\phi\left(w_{1}\right) \geq 1$ if $X^{w_{1}} \in \underline{\mathbf{a}}_{n}$ and $\psi\left(w_{2}\right) \geq 1$ if $Y^{w_{2}} \in \underline{\mathbf{b}}_{n}$, but $\phi(u+e) \leq \alpha / n$ and $\psi(v+f) \leq \beta / n$. Therefore we have $\phi(u+e)+\psi(v+f) \leq \gamma / n$.

If $X^{w_{1}} Y^{w_{2}} \in p^{-1} \underline{\mathbf{a}}_{i} \cdot q^{-1} \underline{\mathbf{b}}_{m-i}$, since $\underline{\mathbf{a}}_{i}^{n / i} \subseteq \underline{\mathbf{a}}_{n}$ and $\underline{\mathbf{b}}_{i}^{n / i} \subseteq \underline{\mathbf{b}}_{n}$, we get $(n / i) w_{1} \in$ $P_{\underline{\mathbf{a}}_{n}}$ and $(n /(m-i)) w_{2} \in P_{\underline{\mathbf{b}}_{n}}$. We deduce that $\phi\left(w_{1}\right)+\psi\left(w_{2}\right) \geq i / n+(m-i) / n=$ $m / n$. If $X^{w_{1}} Y^{w_{2}} \in p^{-1} \underline{\mathbf{a}}_{m}$, then $\phi\left(w_{1}\right) \geq m / n$ and $\psi\left(w_{2}\right) \geq 0$ and we have analogous inequalities if $X^{w_{1}} Y^{w_{2}} \in q^{-1} \underline{\mathbf{b}}_{m}$.

This shows that the linear map $\rho: \mathbb{R}^{r} \times \mathbb{R}^{s} \longrightarrow \mathbb{R}$, given by $\rho\left(w_{1}, w_{2}\right)=$ $\phi\left(w_{1}\right)+\psi\left(w_{2}\right)$ has the property that $(n / m) \rho\left(w_{1}, w_{2}\right) \geq 1$ if

$$
X^{w_{1}} Y^{w_{2}} \in p^{-1} \underline{\mathbf{a}}_{m}+\sum_{i=1}^{m-1} p^{-1} \underline{\mathbf{a}}_{i} \cdot q^{-1} \underline{\mathbf{b}}_{m-i}+q^{-1} \underline{\mathbf{b}}_{m} .
$$

Since we have

$$
X^{u} Y^{v} \in \mathcal{I}\left(\mathbb{A}^{r} \times \mathbb{A}^{s}, \gamma / m \cdot\left(p^{-1} \underline{\mathbf{a}}_{m}+\sum_{i=1}^{m-1} p^{-1} \underline{\mathbf{a}}_{i} \cdot q^{-1} \underline{\mathbf{b}}_{m-i}+q^{-1} \underline{\mathbf{b}}_{m}\right)\right),
$$

one more application of Howald's theorem gives $(n / m)(\phi(u+e)+\psi(v+f))>$ $\gamma / m$, a contradiction.

We can give now the proof of the general case.

Proof of Theorem 1.1. Let $f: X^{\prime} \longrightarrow X$ and $g: Y^{\prime} \longrightarrow Y$ be log resolutions for all pairs $\left(X, \underline{\mathbf{a}}_{i}\right)$ and $\left(Y, \underline{\mathbf{b}}_{i}\right)$, and also for $\left(X, \sum_{i} \underline{\mathbf{a}}_{i}\right)$ and $\left(Y, \sum_{i} \underline{\mathbf{b}}_{i}\right)$, respectively.

Let $\underline{\mathbf{a}}_{i}^{\prime}=f^{-1} \underline{\mathbf{a}}_{i}$ and $\underline{\mathbf{b}}_{i}^{\prime}=g^{-1} \underline{\mathbf{b}}_{i}$. If $p^{\prime}: X^{\prime} \times Y^{\prime} \longrightarrow X^{\prime}$ and $q^{\prime}: X^{\prime} \times Y^{\prime} \longrightarrow Y^{\prime}$ are the canonical projections, then we use the notation

$$
\begin{gathered}
\underline{\mathbf{r}}=p^{-1} \underline{\mathbf{a}}_{m}+\sum_{i=1}^{m-1} p^{-1} \underline{\mathbf{a}}_{i} \cdot q^{-1} \underline{\mathbf{b}}_{m-i}+q^{-1} \underline{\mathbf{b}}_{m}, \\
\underline{\mathbf{r}}^{\prime}=p^{\prime-1} \underline{\mathbf{a}}_{m}^{\prime}+\sum_{i=1}^{m-1} p^{\prime-1} \underline{\mathbf{a}}_{i}^{\prime} \cdot q^{\prime-1} \underline{\mathbf{b}}_{m-i}^{\prime}+q^{\prime-1} \underline{\mathbf{b}}_{m}^{\prime} .
\end{gathered}
$$

If $h=(f, g): X^{\prime} \times Y^{\prime} \longrightarrow X \times Y$, then $\underline{\mathbf{r}}^{\prime}=h^{-1} \underline{\mathbf{r}}$.

Suppose first that the assertion of the theorem is true for $X^{\prime}, Y^{\prime},\left\{\underline{\mathbf{a}}_{i}^{\prime}\right\}_{i}$ and $\left\{\underline{\mathbf{b}}_{i}^{\prime}\right\}_{i}$. The change of variable formula for multiplier ideals gives

$$
\mathcal{I}(X \times Y, \gamma / m \cdot \underline{\mathbf{r}})=h_{*}\left(\mathcal{I}\left(X^{\prime} \times Y^{\prime}, \gamma / m \cdot \underline{\mathbf{r}}^{\prime}\right) \otimes \mathcal{O}\left(K_{X^{\prime} \times Y^{\prime} / X \times Y}\right)\right) .
$$

Using also Lemma 1.2 we deduce $\mathcal{I}(X \times Y, \gamma / m \cdot \underline{\mathbf{r}}) \subseteq$

$$
\begin{aligned}
& h_{*}\left(\bigcap_{\alpha+\beta=\gamma}\left(p^{\prime-1} \mathcal{I}\left(X^{\prime}, \alpha / n \cdot \underline{\mathbf{a}}_{n}^{\prime}\right)+q^{\prime-1} \mathcal{I}\left(Y^{\prime}, \beta / n \cdot \underline{\mathbf{b}}_{n}^{\prime}\right)\right) \otimes \mathcal{O}\left(K_{X^{\prime} \times Y^{\prime} / X \times Y}\right)\right) \\
& =\bigcap_{\alpha+\beta=\gamma} h_{*}\left(\left(p^{\prime-1} \mathcal{I}\left(X^{\prime}, \alpha / n \cdot \underline{\mathbf{a}}_{n}^{\prime}\right)+q^{\prime-1} \mathcal{I}\left(Y^{\prime}, \beta / n \cdot \underline{\mathbf{b}}_{n}^{\prime}\right)\right) \otimes \mathcal{O}\left(K_{X^{\prime} \times Y^{\prime} / X \times Y}\right)\right) .
\end{aligned}
$$


Indeed, the sets $\left\{\mathcal{I}\left(X^{\prime}, \alpha \cdot \underline{\mathbf{a}}_{n}^{\prime}\right) \mid \alpha \leq \gamma / n\right\}$ and $\left\{\mathcal{I}\left(Y^{\prime}, \beta \cdot \underline{\mathbf{a}}_{n}^{\prime}\right) \mid \beta \leq \gamma / n\right\}$ are finite, so the above intersection has finitely many distinct terms, and therefore commutes with push-forward.

Note that if $\mathcal{F}^{\prime}, \mathcal{F}^{\prime \prime} \subseteq \mathcal{F}$ are quasicoherent subsheaves of $\mathcal{O}_{X^{\prime} \times Y^{\prime}}$-modules and if $R^{1} h_{*}\left(\mathcal{F}^{\prime} \cap \mathcal{F}^{\prime \prime}\right)=0$, then we have $h_{*}\left(\mathcal{F}^{\prime}+\mathcal{F}^{\prime \prime}\right)=h_{*} \mathcal{F}^{\prime}+h_{*} \mathcal{F}^{\prime \prime}$. Indeed, this follows by applying $h_{*}$ to the exact sequence

$$
0 \longrightarrow \mathcal{F}^{\prime} \cap \mathcal{F}^{\prime \prime} \longrightarrow \mathcal{F}^{\prime} \oplus \mathcal{F}^{\prime \prime} \longrightarrow \mathcal{F}^{\prime}+\mathcal{F}^{\prime \prime} \longrightarrow 0 .
$$

Note that

$$
\begin{aligned}
& R^{1} h_{*}\left(\left(p^{\prime-1} \mathcal{I}\left(X^{\prime}, \alpha / n \cdot \underline{\mathbf{a}}_{n}^{\prime}\right)\right.\right.\left.\left.\cap{q^{\prime}}^{-1} \mathcal{I}\left(Y^{\prime}, \beta / n \cdot \underline{\mathbf{b}}_{n}^{\prime}\right)\right) \otimes \mathcal{O}\left(K_{X^{\prime} \times Y^{\prime} / X \times Y}\right)\right) \\
&=R^{1} h_{*}\left(p^{\prime-1}\left(\mathcal{I}\left(X^{\prime}, \alpha / n \cdot \underline{\mathbf{a}}_{n}^{\prime}\right) \otimes \mathcal{O}\left(K_{X^{\prime} / X}\right)\right)\right. \\
&\left.\otimes q^{\prime-1}\left(\mathcal{I}\left(Y^{\prime}, \beta / n \cdot \underline{\mathbf{b}}_{n}^{\prime}\right) \otimes \mathcal{O}\left(K_{Y^{\prime} / Y}\right)\right)\right)
\end{aligned}
$$

vanishes. This follows by applying the Künneth formula and the Local Vanishing Theorem (see Ein 1.4) which gives $R^{1} f_{*}\left(\mathcal{I}\left(X^{\prime}, \alpha / n \cdot \underline{\mathbf{a}}_{n}^{\prime}\right) \otimes \mathcal{O}\left(K_{X^{\prime} / X}\right)\right)=0$ and $R^{1} g_{*}\left(\mathcal{I}\left(Y^{\prime}, \beta / n \cdot \underline{\mathbf{b}}_{n}^{\prime}\right) \otimes \mathcal{O}\left(K_{Y^{\prime} / Y}\right)\right)=0$.

Using the fact that $f_{*}\left(\mathcal{O}\left(K_{X^{\prime} / X}\right)\right)=\mathcal{O}_{X}$ and $g_{*}\left(\mathcal{O}\left(K_{Y^{\prime} / Y}\right)\right)=\mathcal{O}_{Y}$, one more application of the Künneth formula and of the change of variable formula for the multiplier ideals gives

$$
\begin{aligned}
& h_{*}\left(p^{\prime-1} \mathcal{I}\left(X^{\prime}, \alpha / n \cdot \underline{\mathbf{a}}_{n}^{\prime}\right) \otimes \mathcal{O}\left(K_{X^{\prime} \times Y^{\prime} / X \times Y}\right)\right)=p^{-1} \mathcal{I}\left(X, \alpha / n \cdot \underline{\mathbf{a}}_{n}\right), \\
& h_{*}\left(q^{\prime-1} \mathcal{I}\left(Y^{\prime}, \beta / n \cdot \underline{\mathbf{b}}_{n}^{\prime}\right) \otimes \mathcal{O}\left(K_{X^{\prime} \times Y^{\prime} / X \times Y}\right)\right)=q^{-1} \mathcal{I}\left(Y, \beta / n \cdot \underline{\mathbf{b}}_{n}\right) .
\end{aligned}
$$

Putting everything together, we get via Lemma 1.2 the statement of the theorem. To finish the proof, it is therefore enough to consider the case when all $\underline{\mathbf{a}}_{i}$ and $\underline{\mathbf{b}}_{i}$ are ideals defining effective divisors on $X$ and $Y$, respectively, whose union has simple normal crossings.

Since the statement of the theorem is local in $X$ and $Y$, we may assume that we have étale morphisms $\phi: X \longrightarrow \mathbb{A}^{r}$ and $\psi: Y \longrightarrow \mathbb{A}^{s}$ whose images contain the origins in the respective affine spaces, and principal monomial ideals $\underline{\mathbf{a}}_{i}$ and $\underline{\mathbf{b}}_{i}$ such that $\underline{\mathbf{a}}_{i}=\phi^{-1} \underline{\widetilde{\mathbf{a}}}_{i}$ and $\underline{\mathbf{b}}_{i}=\psi^{-1} \underline{\mathbf{b}}_{i}$, for all $i$.

Since $\phi$ and $\psi$ are étale, the hypothesis implies $\underline{\widetilde{\mathbf{a}}}_{i}^{n / i} \subseteq \underline{\widetilde{\mathbf{a}}}_{n}$ and $\underline{\widetilde{\mathbf{b}}}_{i}^{n / i} \subseteq \widetilde{\widetilde{\mathbf{b}}} n$, for all $1 \leq i \leq n$. Moreover, taking multiplier ideals commutes with the pull-back by étale morphisms, so that we can reduce the theorem to the case of monomial ideals, when it follows from Lemma [1.3.

As in [DEL, we can use the Restriction Theorem to deduce from Theorem 1.1 a property of families of ideals on the same variety.

Corollary 1.4. Let $X$ be a smooth variety and $\underline{\mathbf{a}}_{i} \subseteq \mathcal{O}_{X}, \underline{\mathbf{b}}_{i} \subseteq \mathcal{O}_{X}$ nonzero sheaves of $i d e a l s$, with $1 \leq i \leq m$ or $i=n$. If for every $1 \leq i \leq m$, we have $i \mid n$ and $\underline{\mathbf{a}}_{i}^{n / i} \subseteq \underline{\mathbf{a}}_{n}, \underline{\mathbf{b}}_{i}^{n / i} \subseteq \underline{\mathbf{b}}_{n}$, then for every $\gamma \in \mathbb{Q}_{+}$, we get

$$
\mathcal{I}\left(X, \gamma / m \cdot\left(\underline{\mathbf{a}}_{m}+\sum_{i=1}^{m-1} \underline{\mathbf{a}}_{i} \cdot \underline{\mathbf{b}}_{m-i}+\underline{\mathbf{b}}_{m}\right)\right) \subseteq \sum_{\alpha+\beta=\gamma} \mathcal{I}\left(X, \alpha / n \cdot \underline{\mathbf{a}}_{n}\right) \cdot \mathcal{I}\left(X, \beta / n \cdot \underline{\mathbf{b}}_{n}\right) .
$$


Proof. Consider the diagonal embedding $X \hookrightarrow X \times X$. If $p: X \times X \longrightarrow X$ and $q: X \times X \hookrightarrow X$ are the projections on the first and, respectively, the second component, let

$$
\underline{\mathbf{r}}=p^{-1} \underline{\mathbf{a}}_{m}+\sum_{i=1}^{m} p^{-1} \underline{\mathbf{a}}_{i} \cdot q^{-1} \underline{\mathbf{b}}_{m-i}+q^{-1} \underline{\mathbf{b}}_{m}
$$

Note that we have $\underline{\mathbf{r}} \cdot \mathcal{O}_{X}=\underline{\mathbf{a}}_{m}+\sum_{i=1}^{m-1} \underline{\mathbf{a}}_{i} \cdot \underline{\mathbf{b}}_{m-i}+\underline{\mathbf{b}}_{m}$.

We clearly have $X \nsubseteq V(\underline{\mathbf{r}})$, so that by the Restriction Theorem (see [Ein] 2.1) we deduce

$$
\mathcal{I}\left(X, \gamma / m \cdot\left(\underline{\mathbf{a}}_{m}+\sum_{i=1}^{m-1} \underline{\mathbf{a}}_{i} \cdot \underline{\mathbf{b}}_{m-i}+\underline{\mathbf{b}}_{m}\right)\right) \subseteq \mathcal{I}(X \times X, \gamma / m \cdot \underline{\mathbf{r}}) \cdot \mathcal{O}_{X} .
$$

On the other hand, Theorem 1.1 gives

$$
\begin{aligned}
\mathcal{I}(X \times X, \gamma / m \cdot \underline{\mathbf{r}}) \cdot \mathcal{O}_{X} \subseteq\left(\sum_{\alpha+\beta=\gamma} p^{-1} \mathcal{I}\left(X, \alpha / n \cdot \underline{\mathbf{a}}_{n}\right) \cdot q^{-1} \mathcal{I}\left(X, \beta / n \cdot \underline{\mathbf{b}}_{n}\right)\right) \cdot \mathcal{O}_{X} \\
=\sum_{\alpha+\beta=\gamma} \mathcal{I}\left(X, \alpha / n \cdot \underline{\mathbf{a}}_{n}\right) \cdot \mathcal{I}\left(X, \beta / n \cdot \underline{\mathbf{b}}_{n}\right) .
\end{aligned}
$$

The above inclusions imply the statement of the corollary.

We can give now the proofs of the statements announced in the Introduction.

Proof of Theorem 0.2. Using the fact that

$$
\mathcal{I}\left(X, \alpha \cdot\left\|\underline{\mathbf{a}}_{p}\right\|\right)=\mathcal{I}\left(X, p \alpha \cdot\left\|\underline{\mathbf{a}}_{1}\right\|\right)
$$

and similar equalities for $\underline{\mathbf{b}}_{\bullet}$ and $\underline{\mathbf{c}}_{\bullet}$, we reduce immediately to the case $p=1$. By definition, we have to prove that for every $m \geq 1$, we have

$$
\mathcal{I}\left(X, \gamma / m \cdot \underline{\mathbf{c}}_{m}\right) \subseteq \sum_{\alpha+\beta=\gamma} \mathcal{I}\left(X, \alpha \cdot\left\|\underline{\mathbf{a}}_{1}\right\|\right) \cdot \mathcal{I}\left(X, \beta \cdot\left\|\underline{\mathbf{b}}_{1}\right\|\right) .
$$

If $n$ is a positive integer such that for every $1 \leq i \leq m$ we have $i \mid n$, then we can apply Corollary 1.4 to get

$$
\mathcal{I}\left(X, \gamma / m \cdot \underline{\mathbf{c}}_{m}\right) \subseteq \sum_{\alpha+\beta=\gamma} \mathcal{I}\left(X, \alpha / n \cdot \underline{\mathbf{a}}_{n}\right) \cdot \mathcal{I}\left(X, \beta / b \cdot \underline{\mathbf{b}}_{n}\right) .
$$

On the other hand we have by definition $\mathcal{I}\left(X, \alpha / n \cdot \underline{\mathbf{a}}_{n}\right) \subseteq \mathcal{I}\left(X, \alpha \cdot\left\|\underline{\mathbf{a}}_{1}\right\|\right)$ and a similar inclusion for $\underline{\mathbf{b}}$. This proves the statement of the corollary.

Proof of Theorem 0.1 . This is precisely the statement of Corollary 1.4 in the case $m=n=1$.

We give now the proof of Theorem 0.3 . Recall that it says that in the particular case $m=n=1$, the inclusion in Theorem 1.1 becomes equality.

Proof of Theorem 0.3. The proof of Theorem[1.1 applies word by word in this case if we know that we have equality for monomial ideals. Therefore we may assume that $X=\mathbb{A}^{r}, Y=\mathbb{A}^{s}$ and that $\underline{\mathbf{a}} \subseteq \mathbb{C}[X]=\mathbb{C}\left[X_{1}, \ldots, X_{r}\right]$ and $\underline{\mathbf{b}} \subseteq \mathbb{C}[Y]=$ $\mathbb{C}\left[Y_{1}, \ldots, Y_{s}\right]$ are monomial ideals. We have to prove that if $\alpha+\beta=\gamma$, then

$$
p^{-1} \mathcal{I}\left(\mathbb{A}^{r}, \alpha \cdot \underline{\mathbf{a}}\right) \cdot q^{-1} \mathcal{I}\left(\mathbb{A}^{s}, \beta \cdot \underline{\mathbf{b}}\right) \subseteq \mathcal{I}\left(\mathbb{A}^{r} \times \mathbb{A}^{s}, \gamma \cdot\left(p^{-1} \underline{\mathbf{a}}+q^{-1} \underline{\mathbf{b}}\right)\right) \text {. }
$$


If $\underline{\mathbf{a}}=\mathbb{C}[X]$ or $\underline{\mathbf{b}}=\mathbb{C}[Y]$, then the right-hand side of the above inclusion is $\mathbb{C}[X, Y]$, and the statement is trivial. Suppose therefore that we are in none of these cases. Moreover, if $\alpha=0$, then it is easy to see from the definition of multiplier ideals that $q^{-1} \mathcal{I}\left(\mathbb{A}^{s}, \gamma \cdot \underline{\mathbf{b}}\right)=\mathcal{I}\left(\mathbb{A}^{r} \times \mathbb{A}^{s}, \gamma \cdot q^{-1} \underline{\mathbf{b}}\right.$ ) (note that Proposition 2.2 in [DEL] and its extension to the case of ideals give a more general statement). In this case we get the above inclusion since $\mathcal{I}\left(\mathbb{A}^{r} \times \mathbb{A}^{s}, \gamma \cdot q^{-1} \underline{\mathbf{b}}\right) \subseteq \mathcal{I}\left(\mathbb{A}^{r} \times \mathbb{A}^{s}, \gamma \cdot\left(p^{-1} \underline{\mathbf{a}}+q^{-1} \underline{\mathbf{b}}\right)\right)$. Therefore we may assume that $\alpha>0$, and by symmetry, also that $\beta>0$.

We use again the description in [Ho] for multiplier ideals of monomial ideals. First, this description shows that these ideals are generated by monomials.

Suppose that we have $X^{u} Y^{v} \in p^{-1} \mathcal{I}\left(\mathbb{A}^{r}, \alpha \cdot \underline{\mathbf{a}}\right) \cdot q^{-1} \mathcal{I}\left(\mathbb{A}^{s}, \beta \cdot \underline{\mathbf{b}}\right)$. Let $\phi: \mathbb{R}^{r} \times$ $\mathbb{R}^{s} \longrightarrow \mathbb{R}$ be a linear map such that $\phi\left(w_{1}, 0\right) \geq 1$ if $X^{w_{1}} \in \underline{\mathbf{a}}$ and $\phi\left(0, w_{2}\right) \geq 1$ if $Y^{w_{2}} \in \underline{\mathbf{b}}$. By $[\mathrm{Ho}$, it is enough to prove that for every such $\phi$ we have $\phi(u, v)>\gamma$.

On the other hand, since $X^{u} \in \mathcal{I}\left(\mathbb{A}^{r}, \alpha \cdot \underline{\mathbf{a}}\right)$ we get $\phi(u, 0)>\alpha$. Similarly, since $Y^{v} \in \mathcal{I}\left(\mathbb{A}^{s}, \beta \cdot \underline{\mathbf{b}}\right)$ we get $\phi(0, v)>\beta$. This implies that $\phi(u, v)>\gamma$.

Remark 1.5. If we make the convention that $\mathcal{I}(X, \gamma \cdot(0))$ is equal to $\mathcal{O}_{X}$ if $\gamma=0$ and (0) otherwise, then the formula in Theorem 0.3 is still valid if $\underline{\mathbf{a}}=(0)$ or $\underline{\mathbf{b}}=(0)$. Indeed, if for example $\underline{\mathbf{a}}=(0)$, then the formula in Theorem 0.3 says that

$$
\mathcal{I}\left(X \times Y, \gamma \cdot q^{-1} \underline{\mathbf{b}}\right)=q^{-1} \mathcal{I}(Y, \gamma \cdot \underline{\mathbf{b}}) .
$$

But as we have mentioned, this is a particular case of the results in DEL].

\section{INVARIANCE OF MULTIPLIER IDEALS}

We start by showing that Theorem 0.1 can be used to approximate arbitrary multiplier ideals by multiplier ideals corresponding to zero dimensional ideals.

Fix a smooth variety $X$ with $\operatorname{dim} X=n$ and $\underline{\mathbf{a}} \subseteq \mathcal{O}_{X}$ a nonzero sheaf of ideals on $X$. For a (closed) point $x \in X$, let $\underline{\mathbf{m}}_{x} \subseteq \mathcal{O}_{x}$ be the ideal defining that point. If $l \leq 0$ is an integer, we put $\underline{\mathbf{m}}_{x}^{l}=\mathcal{O}_{X}$. Recall that [.] denotes the integral part function.

Proposition 2.1. With the above notation, for every $x \in X$, every integer $p \geq 1$, and every $\gamma \in \mathbb{Q}_{+}, \epsilon \in \mathbb{Q}_{+}^{*}$, we have

$$
\mathcal{I}\left(X,(\gamma+\epsilon) \cdot\left(\underline{\mathbf{a}}+\underline{\mathbf{m}}_{x}^{p}\right)\right) \subseteq \mathcal{I}(X, \gamma \cdot \underline{\mathbf{a}})+\underline{\mathbf{m}}_{x}^{[p \epsilon]-n+1} .
$$

Proof. We apply Theorem 0.1 to the sheaves of ideals $\underline{\mathbf{a}}$ and $\underline{\mathbf{m}}_{x}^{p}$ and to $\gamma+\epsilon$. Note that the multiplier ideals of $\underline{\mathbf{m}}_{x}^{p}$ are given by

$$
\mathcal{I}\left(X, \beta \cdot \underline{\mathbf{m}}_{x}^{p}\right)=\underline{\mathbf{m}}_{x}^{[p \beta]-n+1},
$$

for every $p \geq 1$. We therefore obtain

$$
\begin{aligned}
& \mathcal{I}\left(X,(\gamma+\epsilon) \cdot\left(\underline{\mathbf{a}}+\underline{\mathbf{m}}_{x}^{p}\right)\right) \subseteq \sum_{\alpha+\beta=\gamma+\epsilon} \mathcal{I}(X, \alpha \cdot \underline{\mathbf{a}}) \cdot \mathcal{I}\left(X, \beta \cdot \underline{\mathbf{m}}_{x}^{p}\right) \\
& \subseteq \mathcal{I}(X, \gamma \cdot \underline{\mathbf{a}})+\sum_{\beta>\epsilon} \mathcal{I}\left(X, \beta \cdot \underline{\mathbf{m}}_{x}^{p}\right)=\mathcal{I}(X, \gamma \cdot \underline{\mathbf{a}})+\mathcal{I}\left(X, \epsilon \cdot \underline{\mathbf{m}}_{x}^{p}\right) .
\end{aligned}
$$

The statement of the proposition now follows from this and the formula for the multiplier ideals of $\underline{\mathbf{m}}_{x}^{p}$. 
Remark 2.2. With the notation in the above proposition, let $I=\mathcal{I}(X, \gamma \cdot \underline{\mathbf{a}})$. By the semicontinuity property of multiplier ideals with respect to the parameter, we can find $\epsilon \in \mathbb{Q}_{+}^{*}$ such that $\mathcal{I}(X,(\gamma+\epsilon) \cdot \underline{\mathbf{a}})=I$. We deduce from this and Proposition 2.1 that

$$
I+\underline{\mathbf{m}}_{x}^{[p \gamma+p \epsilon]-n+1} \subseteq \mathcal{I}\left(X,(\gamma+\epsilon) \cdot\left(\underline{\mathbf{a}}+\underline{\mathbf{m}}_{x}^{p}\right)\right) \subseteq I+\underline{\mathbf{m}}_{x}^{[p \epsilon]-n+1} .
$$

Since multiplier ideals are integrally closed, we deduce that the integral closure of $I+\underline{\mathbf{m}}_{x}^{[p \gamma+p \epsilon]-n+1}$ is contained in $I+\underline{\mathbf{m}}_{x}^{[p \epsilon]-n+1}$.

As pointed to us by L. Ein and R. Lazarsfeld, this can be considered as an effective version of a theorem of Delfino and Swanson (see [DS]) for the case when $I$ is a multiplier ideal (note however that the result in [DS] holds in arbitrary excellent rings).

We first apply this to study the relation between multiplier ideals on $X$ and $Y$, when $X$ is a subvariety of $Y$. We use the convention that if $\underline{\mathbf{a}} \subseteq \mathcal{O}_{X}$ is a nonzero sheaf of ideals on a smooth variety $X$, then $\mathcal{I}(X, \gamma \cdot \underline{\mathbf{a}})=\mathcal{O}_{X}$, for every $\gamma<0$.

Proposition 2.3. Let $Y$ be a smooth variety and $X \subset Y$ a closed smooth subvariety, with $\operatorname{codim}(X / Y)=r$. If $\underline{\mathbf{a}}_{X} \subset \mathcal{O}_{Y}$ is the sheaf of ideals defining $X$ and $\underline{\mathbf{b}}$ is a sheaf of ideals such that $\underline{\mathbf{a}}_{X} \subset \underline{\mathbf{b}}$ and $\underline{\mathbf{a}}_{X} \neq \underline{\mathbf{b}}$, then

$$
\mathcal{I}\left(X, \gamma \cdot \underline{\mathbf{b}} / \underline{\mathbf{a}}_{X}\right)=\mathcal{I}(Y,(\gamma+r) \cdot \underline{\mathbf{b}}) \cdot \mathcal{O}_{X},
$$

for every $\gamma \in \mathbb{Q}$.

Proof. We consider first the case when

$$
X=\mathbb{A}^{n} \hookrightarrow Y=X \times \mathbb{A}^{r},
$$

is defined by the vanishing of the last $r$ coordinates. Let $p: X \times \mathbb{A}^{r} \longrightarrow X$ and $q: X \times \mathbb{A}^{r} \longrightarrow \mathbb{A}^{r}$ be the canonical projections.

If $\underline{\mathbf{m}}_{0}$ is the ideal defining the origin in $\mathbb{A}^{r}$, then $\underline{\mathbf{a}}_{X}=q^{-1} \underline{\mathbf{m}}_{0}$. Moreover, we have $\underline{\mathbf{b}}=p^{-1}\left(\underline{\mathbf{b}} / \underline{\mathbf{a}}_{X}\right)+q^{-1} \underline{\mathbf{m}}_{0}$. We know that $\mathcal{I}\left(\mathbb{A}^{r}, \beta \cdot \underline{\mathbf{m}}_{0}\right)$ is equal to $\underline{\mathbf{m}}_{0}^{[\beta]-r+1}$, if $\beta \geq r-1$, and it is equal with $\mathcal{O}_{\mathbb{A}^{r}}$, otherwise.

We may assume that $\gamma+r \geq 0$ because otherwise the statement of the proposition is trivial. Theorem 0.3 gives

$$
\mathcal{I}(Y,(\gamma+r) \cdot \underline{\mathbf{b}})=\sum_{\alpha+\beta=\gamma+r} p^{-1} \mathcal{I}\left(X, \alpha \cdot \underline{\mathbf{b}} / \underline{\mathbf{a}}_{X}\right) \cdot q^{-1} \mathcal{I}\left(\mathbb{A}^{r}, \beta \cdot \underline{\mathbf{m}}_{0}\right) .
$$

Since we have $q^{-1} \mathcal{I}\left(\mathbb{A}^{r}, \beta \cdot \underline{\mathbf{m}}_{0}\right) \cdot \mathcal{O}_{X}=(0)$ if $\beta \geq r$, we deduce that

$$
\mathcal{I}(Y,(\gamma+r) \cdot \underline{\mathbf{b}}) \cdot \mathcal{O}_{X}=\sum_{\gamma<\alpha \leq \gamma+r} \mathcal{I}\left(X, \alpha \cdot \underline{\mathbf{b}} / \underline{\mathbf{a}}_{X}\right)=\mathcal{I}\left(X, \gamma \cdot \underline{\mathbf{b}} / \underline{\mathbf{a}}_{X}\right),
$$

which finishes the proof of this case.

We show now that if $\underline{\mathbf{b}}$ is a zero dimensional ideal, then we can reduce the statement to the above case. Since the statement is local, we may assume that $\operatorname{Supp}\left(\mathcal{O}_{Y} / \underline{\mathbf{b}}\right)=\{x\}$, for some point $x \in X$ and it is enough to check the equality in the proposition in an open neighbourhood of $x$. Therefore we may assume that there is an étale morphism $\phi: Y \longrightarrow \mathbb{A}^{n+r}$ with $\phi(x)=0$ such that $X=\phi^{-1}\left(\mathbb{A}^{n}\right)$. Here we view $\mathbb{A}^{n}$ embedded in $\mathbb{A}^{n+r}$ as before.

Note that $\phi$ induces an isomorphism between the completions of the local rings of $Y$ and $\mathbb{A}^{n+r}$ at $x$ and 0 , respectively. But $\operatorname{dim}\left(\mathcal{O}_{Y} / \underline{\mathbf{b}}\right)_{x}=0$, so that there is an ideal $\underline{\mathbf{b}}^{\prime} \subset \mathcal{O}_{\mathbb{A}^{n+r}}$, such that $\underline{\mathbf{b}}=\phi^{-1} \underline{\mathbf{b}}^{\prime}$. Since construction of multiplier ideals 
commutes with pull-back by étale morphisms, we deduce the proposition in the case of zero dimensional ideals from the case we have already proved.

To finish the proof of the proposition, we show how to deduce the general case from that of zero dimensional ideals. Obviously it is enough to prove that for every $x \in X$ we have equality after localizing at $x: I \cdot \mathcal{O}_{X, x}=J \cdot \mathcal{O}_{X, x}$, where $I:=\mathcal{I}\left(X, \gamma \cdot \underline{\mathbf{b}} / \underline{\mathbf{a}}_{X}\right)$ and $J:=\mathcal{I}(Y,(\gamma+r) \cdot \underline{\mathbf{b}}) \cdot \mathcal{O}_{X}$.

We fix $\epsilon \in \mathbb{Q}_{+}^{*}$ such that $\mathcal{I}\left(X, \gamma \cdot \underline{\mathbf{b}} / \underline{\mathbf{a}}_{X}\right)=\mathcal{I}\left(X,(\gamma+\epsilon) \cdot \underline{\mathbf{b}} / \underline{\mathbf{a}}_{X}\right)$ and $\mathcal{I}(Y,(\gamma+r) \cdot \underline{\mathbf{b}})=\mathcal{I}(Y,(\gamma+r+\epsilon) \cdot \underline{\mathbf{b}})$. Let $\underline{\mathbf{m}}_{x}$ be the ideal of the point $x$ in $Y$.

Using Proposition 2.1 and the fact that we know the statement for the zero dimensional ideal $\underline{\mathbf{b}}+\underline{\mathbf{m}}_{x}^{p}$, for every $p \geq 1$, we get

$$
\begin{gathered}
I \subseteq \mathcal{I}\left(X,(\gamma+\epsilon) \cdot\left(\underline{\mathbf{b}}+\underline{\mathbf{m}}_{x}^{p}\right) / \underline{\mathbf{a}}_{X}\right)=\mathcal{I}\left(Y,(\gamma+\epsilon+r) \cdot\left(\underline{\mathbf{b}}+\underline{\mathbf{m}}_{x}^{p}\right)\right) \cdot \mathcal{O}_{X} \\
\subseteq J+\left(\underline{\mathbf{m}}_{x} / \underline{\mathbf{a}}_{X}\right)^{[p \epsilon]-\operatorname{dim} Y+1} .
\end{gathered}
$$

Since this is true for every $p \geq 1$, Krull's Intersection Theorem gives the inclusion $I \cdot \mathcal{O}_{X, x} \subseteq J \cdot \mathcal{O}_{X, x}$.

The reverse inclusion follows similarly:

$$
\begin{gathered}
J \subseteq \mathcal{I}\left(Y,(\gamma+\epsilon+r) \cdot\left(\underline{\mathbf{b}}+\underline{\mathbf{m}}_{x}^{p}\right)\right) \cdot \mathcal{O}_{X} \\
=\mathcal{I}\left(X,(\gamma+\epsilon) \cdot\left(\underline{\mathbf{b}}+\underline{\mathbf{m}}_{x}^{p}\right) / \underline{\mathbf{a}}_{X}\right) \subseteq J+\left(\underline{\mathbf{m}}_{x} / \underline{\mathbf{a}}_{X}\right)^{[p \epsilon]-\operatorname{dim} X+1},
\end{gathered}
$$

for every $p \geq 1$, and we again apply Krull's Intersection Theorem. This completes the proof of the proposition.

We now compare multiplier ideals corresponding to a scheme in two different arbitrary embeddings. More precisely, we show that the restrictions of the multiplier ideals of $\underline{\mathbf{a}}$ to the subscheme defined by $\underline{\mathbf{a}}$ depend only on this subscheme (they do not depend on the particular embedding into a smooth variety).

Proposition 2.4. If $X_{1}$ and $X_{2}$ are smooth varieties and $\underline{\mathbf{a}}_{1} \subseteq \mathcal{O}_{X_{1}}, \underline{\mathbf{a}}_{2} \subseteq \mathcal{O}_{X_{2}}$ are nonzero sheaves of ideals, then every isomorphism $\phi: Y_{1}=V\left(\underline{\mathbf{a}}_{1}\right) \longrightarrow Y_{2}=V\left(\underline{\mathbf{a}}_{2}\right)$ induces isomorphisms of ideals

$$
\mathcal{I}\left(X_{1},\left(\gamma+\operatorname{dim} X_{1}\right) \cdot \underline{\mathbf{a}}_{1}\right) \cdot \mathcal{O}_{Y_{1}} \simeq \mathcal{I}\left(X_{2},\left(\gamma+\operatorname{dim} X_{2}\right) \cdot \underline{\mathbf{a}}_{2}\right) \cdot \mathcal{O}_{Y_{2}},
$$

for every $\gamma \in \mathbb{Q}$.

Proof. If for example $\operatorname{dim} X_{1}=\operatorname{dim} X_{2}+s$, with $s \geq 1$, by replacing $X_{2}$ with $X_{2} \times$ $\mathbb{A}^{s}$ and applying Proposition 2.3, we reduce ourselves to the case when $\operatorname{dim} X_{1}=$ $\operatorname{dim} X_{2}=n$ and in this case it is enough to prove that for every $\gamma \in \mathbb{Q}_{+}$, we get an induced isomorphism:

$$
\mathcal{I}\left(X_{1}, \gamma \cdot \underline{\mathbf{a}}_{1}\right) \cdot \mathcal{O}_{Y_{1}} \simeq \mathcal{I}\left(X_{2}, \gamma \cdot \underline{\mathbf{a}}_{2}\right) \cdot \mathcal{O}_{Y_{2}} .
$$

As in the proof of Proposition 2.3. we first prove the case $\operatorname{dim} Y_{1}=\operatorname{dim} Y_{2}=0$. To simplify the notation, whenever there is no danger of confusion, we will identify $Y_{1}$ with $Y_{2}$ via $\phi$ and denote it by $Y$. We may clearly assume that the support of $Y$ consists of only one point $y \in Y$.

Let $r=\operatorname{dim} T_{y} Y$. We pick a regular system of parameters $x_{1}, \ldots, x_{n}$ for $X_{1}$ around $y$ such that $x_{r+1}, \ldots, x_{n}$ are in the ideal of $Y_{1}$. After restricting to a suitable open neighbourhood of $y$, this induces an étale morphism $\psi_{1}: X_{1} \longrightarrow \mathbb{A}^{n}$ such that $Y_{1} \subseteq \psi_{1}^{-1}\left(\mathbb{A}^{r}\right)$, where $\mathbb{A}^{r} \subseteq \mathbb{A}^{n}$ is defined by the vanishing of the last $n-r$ 
coordinates. Moreover, since $\operatorname{dim} Y=0$, there is a subscheme $Y_{1}^{\prime} \subseteq \mathbb{A}^{r}$ such that $Y_{1}=\psi_{1}^{-1}\left(Y_{1}^{\prime}\right)$ and $\psi_{1}$ induces an isomorphism $Y_{1} \simeq Y_{1}^{\prime}$.

We get a similar morphism $\psi_{2}: X_{2} \longrightarrow \mathbb{A}^{n}$ with analogous properties. Using the fact that construction of multiplier ideals commutes with pull-back by étale morphisms we reduce the equality in equation (7) to the case when $X_{1}$ and $X_{2}$ are both affine spaces. Moreover, using Proposition 2.3, we see that we may assume that $r=n$.

In this case the isomorphism $\phi: Y_{1} \longrightarrow Y_{2}$ can be lifted to a local ring homomorphism

$$
\tilde{\phi}: \mathcal{O}_{X_{2}, y} \longrightarrow \mathcal{O}_{X_{1}, y}
$$

Since $T_{y} Y_{i}=T_{y} X_{i}$ for $i=1,2$, it follows that $\tilde{\phi}$ induces an isomorphism of the corresponding completion rings i.e. it is étale. By restricting further to neighbourhoods of $y$ in $X_{1}$ and $X_{2}$, we may assume that $\tilde{\phi}$ is induced by an étale scheme morphism $X_{1} \longrightarrow X_{2}$. Using the invariance of multiplier ideals under pull-back for étale morphisms one more time, we deduce equation (7) in the zero-dimensional case.

Suppose now that $Y$ has arbitrary dimension. It is enough to prove that for every $y \in Y$, the analogue of equation (17) holds for the images of those two ideals in $\mathcal{O}_{Y, y}$. Let us denote by $\underline{\mathbf{m}}_{1}$ and $\underline{\mathbf{m}}_{2}$ the ideals defining $y$ in $X_{1}$ and $X_{2}$ respectively. It is clear that we get induced isomophisms $\phi_{p}: V\left(\underline{\mathbf{a}}_{1}+\underline{\mathbf{m}}_{1}^{p}\right) \longrightarrow V\left(\underline{\mathbf{a}}_{2}+\underline{\mathbf{m}}_{2}^{p}\right)$, for every $p \geq 1$.

We apply the statement in the case of the zero dimensional schemes $Y_{1}^{p}=$ $V\left(\underline{\mathbf{a}}_{1}+\underline{\mathbf{m}}_{1}^{p}\right)$ and $Y_{2}^{p}=V\left(\underline{\mathbf{a}}_{2}+\underline{\mathbf{m}}_{2}^{p}\right)$. If $\epsilon \in \mathbb{Q}_{+}^{*}$ is such that

$$
I\left(X_{2}, \gamma \cdot \underline{\mathbf{a}}_{2}\right)=\mathcal{I}\left(X_{2},(\gamma+\epsilon) \cdot \underline{\mathbf{a}}_{2}\right),
$$

then applying also Proposition [2.1, we get

$$
\begin{gathered}
\mathcal{I}\left(X_{1}, \gamma \cdot \underline{\mathbf{a}}_{1}\right) \cdot \mathcal{O}_{Y_{1}^{p}} \subseteq \mathcal{I}\left(X_{1},(\gamma+\epsilon) \cdot\left(\underline{\mathbf{a}}_{1}+\underline{\mathbf{m}}_{1}^{p}\right)\right) \cdot \mathcal{O}_{Y_{1}^{p}} \\
\simeq \mathcal{I}\left(X_{2},(\gamma+\epsilon) \cdot\left(\underline{\mathbf{a}}_{2}+\underline{\mathbf{m}}_{2}^{p}\right)\right) \cdot \mathcal{O}_{Y_{2}^{p}} \subseteq \mathcal{I}\left(X_{2}, \gamma \cdot \underline{\mathbf{a}}_{2}\right) \cdot \mathcal{O}_{Y_{2}^{p}}+\underline{\mathbf{m}}_{2}^{[p \epsilon]-n+1} \cdot \mathcal{O}_{Y_{2}^{p}}
\end{gathered}
$$

This shows that the image of $\mathcal{I}\left(X_{1}, \gamma \cdot \underline{\mathbf{a}}_{1}\right) \cdot \mathcal{O}_{Y_{1}, y}$ by the isomorphism induced by $\phi$ is contained in

$$
\bigcap_{q \geq 1}\left(\mathcal{I}\left(X_{2}, \gamma \cdot \underline{\mathbf{a}}_{2}\right) \cdot \mathcal{O}_{Y_{2}, y}+\underline{\mathbf{m}}_{2}^{q} \cdot \mathcal{O}_{Y_{2}, y}\right)
$$

Krull's Intersection Theorem gives now the inclusion " $\subseteq$ " in equation (7). The reverse inclusion follows by symmetry.

Remark 2.5. Recall that the $\log$ canonical threshold of $(X, V(\underline{\mathbf{a}}))$ is given by

$$
c(X, V(\underline{\mathbf{a}}))=\sup \left\{\alpha \in \mathbb{Q}_{+} \mid \mathcal{I}(X, \alpha \cdot \underline{\mathbf{a}})=\mathcal{O}_{X}\right\} .
$$

Since $\mathcal{I}(X, \alpha \cdot \underline{\mathbf{a}})=\mathcal{O}_{X}$ if and only if $\mathcal{I}(X, c \cdot \underline{\mathbf{a}}) \cdot \mathcal{O}_{V(\underline{\mathbf{a}})}=\mathcal{O}_{V(\underline{\mathbf{a}})}$, it follows from Proposition 2.4 that $\operatorname{dim} X-c(X, V(\underline{\mathbf{a}}))$ is independent on the embedding $V(\underline{\mathbf{a}}) \hookrightarrow$ $X$ (see $\underline{\mathrm{Mu}}$ for an intrinsic expression for this difference). In fact, we show below that this is the case with all the jumping numbers of the multiplier ideals of $\underline{\mathbf{a}}$.

If $\underline{\mathbf{a}} \subseteq \mathcal{O}_{X}$ is a nonzero sheaf of ideals, then we say that $\alpha \in \mathbb{Q}$ is a jumping number of $\underline{\mathbf{a}}$ if

$$
\mathcal{I}\left(X, \alpha^{\prime} \cdot \underline{\mathbf{a}}\right) \neq \mathcal{I}(X, \alpha \cdot \underline{\mathbf{a}}),
$$

for every $\alpha^{\prime}<\alpha$. 
Proposition 2.6. With the notation in Proposition 2.4 if there is an isomorphism $\phi: Y_{1} \longrightarrow Y_{2}$, then for every $\gamma \in \mathbb{Q}, \gamma+\operatorname{dim} X_{1}$ is a jumping number for $\underline{\mathbf{a}}_{1}$ if and only if $\gamma+\operatorname{dim} X_{2}$ is a jumping number for $\underline{\mathbf{a}}_{2}$.

Proof. The argument has the same flavor as the one used in Proposition [2.4, so we just sketch it briefly. Again, it is clear that we may assume that $\operatorname{dim} X_{1}=$ $\operatorname{dim} X_{2}=n$. The main point is to use Proposition 2.1 to show that $\alpha$ is not a jumping number for a sheaf of ideals $\underline{\text { a }}$ on a variety $X$ if and only if there is $\epsilon \in \mathbb{Q}_{+}^{*}$ such that for every $x \in X$ and every $p, q \geq 1$, we have

$$
\mathcal{I}\left(X,(\alpha-\epsilon) \cdot\left(\underline{\mathbf{a}}+\underline{\mathbf{m}}_{x}^{p}\right)\right) \subseteq \mathcal{I}\left(X,(\alpha+\epsilon) \cdot\left(\underline{\mathbf{a}}+\underline{\mathbf{m}}_{x}^{q}\right)\right)+\underline{\mathbf{m}}_{x}^{[p \epsilon]-n+1} .
$$

Indeed, first suppose that $\alpha$ is not a jumping number. Then there is $\epsilon \in \mathbb{Q}_{+}^{*}$ such that

$$
\mathcal{I}(X,(\alpha-2 \epsilon) \cdot \underline{\mathbf{a}})=\mathcal{I}(X,(\alpha+\epsilon) \cdot \underline{\mathbf{a}}) .
$$

Proposition 2.1 gives

$$
\begin{gathered}
\mathcal{I}\left(X,(\alpha-\epsilon) \cdot\left(\underline{\mathbf{a}}+\underline{\mathbf{m}}_{x}^{p}\right)\right) \subseteq \mathcal{I}(X,(\alpha-2 \epsilon) \cdot \underline{\mathbf{a}})+\underline{\mathbf{m}}_{x}^{[p \epsilon]-n+1} \\
=\mathcal{I}(X,(\alpha+\epsilon) \cdot \underline{\mathbf{a}})+\underline{\mathbf{m}}_{x}^{[p \epsilon]-n+1} \subseteq \mathcal{I}\left(X,(\alpha+\epsilon) \cdot\left(\underline{\mathbf{a}}+\underline{\mathbf{m}}_{x}^{q}\right)\right)+\underline{\mathbf{m}}_{x}^{[p \epsilon]-n+1},
\end{gathered}
$$

which gives the inclusion in equation (8).

Conversely, if we have equation (8), then Proposition 2.1 gives

$$
\mathcal{I}\left(X,(\alpha-\epsilon) \cdot\left(\underline{\mathbf{a}}+\underline{\mathbf{m}}_{x}^{p}\right)\right) \subseteq \mathcal{I}(X, \alpha \cdot \underline{\mathbf{a}})+\underline{\mathbf{m}}_{x}^{[q \epsilon]-n+1}+\underline{\mathbf{m}}_{x}^{[p \epsilon]-n+1},
$$

for all $q \geq 1$. Krull's Intersection Theorem implies that

$$
\mathcal{I}\left(X,(\alpha-\epsilon) \cdot\left(\underline{\mathbf{a}}+\underline{\mathbf{m}}_{x}^{p}\right)\right) \cdot \mathcal{O}_{X, x} \subseteq \mathcal{I}(X, \alpha \cdot \underline{\mathbf{a}}) \cdot \mathcal{O}_{X, x}+\underline{\mathbf{m}}_{x}^{[p \epsilon]-n+1} \cdot \mathcal{O}_{X, x} .
$$

Since the left-hand side of the above inclusion contains $\mathcal{I}(X,(\alpha-\epsilon) \cdot \underline{\mathbf{a}}) \cdot \mathcal{O}_{X, x}$, one more application of Krull's Intersection Theorem shows that $\alpha$ is not a jumping number.

Since equation (8) is a statement about zero dimensional subschemes, the proof can be concluded with an argument which parallels the one in Proposition 2.4.

\section{REFERENCES}

[DS] Donatella Delfino and Irena Swanson, Integral closure of ideals in excellent local rings, J. Algebra 187 (1997), 422-445. MR 98a:13014

[DEL] Jean-Pierre Demailly, Lawrence Ein and Robert Lazarsfeld, A subadditivity property of multiplier ideals, Michigan Math. J. 48 (2000), 137-156. CMP 2001:03

[Ein] Lawrence Ein, Multiplier ideals, vanishing theorems and applications, in Algebraic geometry, Santa Cruz, 1995, 203-220, volume 62 of Proc. Sympos. Pure Math., Amer. Math. Soc. 1997. MR 98m:14006

[EL] Lawrence Ein and Robert Lazarsfeld, A geometric effective Nullstellensatz, Invent. Math. 137 (1999), no. 2, 427-448. MR 2000j:14028

[ELS] Lawrence Ein, Robert Lazarsfeld, and Karen Smith, Uniform bounds and symbolic powers on smooth varieties, Invent. Math. 144 (2001), 241-252. CMP 2001:11

[Ho] Jason Howald, Multiplier ideals of monomial ideals, Trans. Amer. Math. Soc. 353 (2001), 2665-2671. CMP 2001:11

[Ka1] Yujiro Kawamata, Deformations of canonical singularities, J. Amer. Math. Soc. 12 (1999), no. 1, 85-92. MR 99g:14003

[Ka2] Yujiro Kawamata, On the extension problem of pluricanonical forms, in Algebraic Geometry: Hirzebruch 70 (Warsaw, 1998), Amer. Math. Soc., Providence, RI, 1999, pp. 193-207. MR 2000i: 14053

[La] Robert Lazarsfeld, Multiplier ideals for algebraic geometers, lecture notes available at http://www.math.lsa.umich.edu/〜 rlaz, version of August 2000. 
[Mu] Mircea Mustaţă, Singularities of pairs via jet schemes, preprint 2001, arXiv: math. AG/0102201.

[Siu] Yum-Tong Siu, Invariance of plurigenera, Invent. Math. 134 (1998), no. 3, 661-673. MR 99i:32035

Department of Mathematics, University of California, Berkeley, California 94720 and Institute of Mathematics of The Romanian Academy, Bucharest, Romania

E-mail address: mustata@math.berkeley.edu 